\title{
A fuzzy neural network model to forecast the percent cloud coverage and cloud top temperature maps
}

\author{
Y. Tulunay ${ }^{1}$, E. T. Şenalp ${ }^{2}$, Ş. Ö $z^{3}$, L. I. Dorman ${ }^{4,5}$, E. Tulunay ${ }^{2}$, S. S. Menteş ${ }^{6}$, and M. E. Akcan ${ }^{6}$ \\ ${ }^{1}$ ODTÜ/METU, Dept. of Aerospace Eng., Ankara, Turkey \\ ${ }^{2}$ ODTÜ/METU, Dept. of Electrical and Electronics Eng., Ankara, Turkey \\ ${ }^{3}$ DMI, Turkish State Meteorological Service, Ankara, Turkey \\ ${ }^{4}$ Israel Cosmic Ray and Space Weather Center and Emilio Segre' Observatory affiliated to Tel Aviv University, Technion and \\ Israel Space Agency, Tel Aviv, Israel \\ ${ }^{5}$ Russian Academy of Science, Cosmic Ray Dept. of IZMIRAN, Russia \\ ${ }^{6}$ İTÜ, Dept. of Meteorological Eng., İstanbul, Turkey
}

Received: 20 August 2007 - Revised: 1 September 2008 - Accepted: 29 October 2008 - Published: 5 December 2008

\begin{abstract}
Atmospheric processes are highly nonlinear. A small group at the METU in Ankara has been working on a fuzzy data driven generic model of nonlinear processes. The model developed is called the Middle East Technical University Fuzzy Neural Network Model (METU-FNNM). The METU-FNN-M consists of a Fuzzy Inference System (METU-FIS), a data driven Neural Network module (METU-FNN) of one hidden layer and several neurons, and a mapping module, which employs the Bezier Surface Mapping technique. In this paper, the percent cloud coverage (\% CC) and cloud top temperatures (CTT) are forecast one month ahead of time at 96 grid locations. The probable influence of cosmic rays and sunspot numbers on cloudiness is considered by using the METU-FNN-M.
\end{abstract}

Keywords. Interplanetary physics (Cosmic rays; Energetic particles; Instruments and techniques)

\section{Introduction}

There have been several possible causes of global climate change discussed in the scientific literature (e.g. Svensmark, 2000, and the references therein). One of such possibilities has been the change in solar activity and Cosmic Ray (CR) intensity (Dorman, 2004). Cosmic rays consist of solar and non-solar born energetic particles, which originate from all directions in space. The geomagnetic field influences the $\mathrm{CR}$ flux (CRF). The interaction of geomagnetic field with the CR

Correspondence to: E. T. Şenalp

(senalp@eee.metu.edu.tr) yields some ionization in the lower part of the atmosphere (Palle et al., 2004).

Within the European Science Foundation (ESF) Network: SPECIAL, various atmospheric electrodynamic and electromagnetic processes associated with variable solar output of electromagnetic and corpuscular energies were considered. In particular, the physics based on numerical model of the Earth's atmosphere were studied in order to test the hypothesis on possible links between the Space Weather and the Earth's weather and climate (private communication: M. Fullekrug, Coordinator of the ESF Network: Space Weather and the Earth's Weather and Climate (SPECIAL), Frankfurt, Germany, 2002) (www.esf.org/fileadmin/be_user/ research_areas/PESC/Documents/SPECIAL2Flyer.pdf).

Carslaw et al. (2002) reported that the CR intensity and the Earth's average cloud cover seem to be correlated over one solar cycle. The implication of such a correlation is that the Earth's climate could be affected by changes in percent cloud coverage (\%CC). The occurrence of clouds in the atmosphere is well understood in terms of meteorological parameters, e.g. humidity, temperature, atmospheric dynamics etc. However, there are many properties of clouds influenced by a series of complex, nonlinear microphysical processes (Carslaw et al., 2002). For example, it has been suggested that CR induced ionization may have direct and indirect impacts on cloud microphysics (Todd and Kniveton, 2001).

The magnitude of the direct and indirect Galactic CR cloud interactions in the Near Earth Space remains a controversial issue. In another words, the possible influence of the charged particles on the Earth's weather and climate is subject to a continuing scientific debate. So far, the evidence has

Published by Copernicus Publications on behalf of the European Geosciences Union. 
been based on empirical observations and, to a lesser extent, on quantitative physical modeling of many coupled nonlinear processes and/or on numerical models. For such cases, data driven modeling methods have been recommended to be used in parallel with mathematical modeling approach (e.g. Tulunay, 1991). Highly nonlinear processes in the Near Earth Space are advantageously dealt with using data driven modeling techniques in the Neural Network (NN) approach providing there are representative data (e.g. Tulunay et al., 2004a). Therefore, there is a clear need for observational studies to assess the evidence for the operation of the mechanisms proposed at climate space and time scales (Todd and Kniveton, 2001; Usoskin et al., 2004). While the observations can be interpreted, the observed data are used as input to the data driven models.

The Near Earth Space variability at several different time scales arises from a number of separate factors. The physics of those variations cannot be modeled due to the lack of current information about the parameters of several natural processes.

For example CR are shielded by the magnetosphere to a certain extent, but they can modulate the low level cloud cover through electrically mediated microphysical processes in the clouds. In turn, the cloud cover variability strongly influences weather and climate changes.

CR affects stratosphere, and depending on the amount of the energy, temperature is increased. This event affects the troposphere layer, which has the dynamics of the meteorological and climate related processes. Clouds are formed from the water vapor in the troposphere.

Because of the spatial and temperature differences, the layer width of the troposphere shows considerable decrease at the region in between the equator and the North Pole. In a previous work, it was observed that the CR and the Sun Spot Numbers (SSN) affect the formation of the clouds on mid and high latitudes of Europe (Svensmark, 2000). These are namely the low level clouds, which bring rains.

In our work, we considered a considerably informative spatial region, which covers Europe.

With the state of art on data driven modeling that the authors have gained on NN based models in relation to understanding the Near Earth Space processes since 1990s, this time, they propose a Fuzzy Neural Network Model (METUFNN-M) in order to forecast the percent cloud coverage $(\% \mathrm{CC})$ and cloud top temperature (CTT) maps between geographic coordinates of $\left(22.5^{\circ} \mathrm{N} ; 57.5^{\circ} \mathrm{N}\right)$; and $\left(7.5^{\circ} \mathrm{W}\right.$; $47.5^{\circ} \mathrm{E}$ ) covering a low sunspot number period during the years of 1983 to 2000 .

In this work, we investigated possible influence of the CR and SSN on the variation of upper atmosphere parameters, i.e. \%CC and CTT. The METU-FNN-M is developed to forecast and map these meteorological variables without classifying the clouds (Tulunay et al., 2007).

The METU-FNN-M can be applied on clustering clouds as a future work. There are plenty of works on classification of clouds. Different techniques are applied for clustering process with a variety of input data source.

Rossow et al. (2005) developed a statistical model that associates cloud types, recognizable from satellite measurements, with particular cloud vertical structures. They combined the statistics of cloud layer occurrence from the International Satellite Cloud Climatology Project (ISCCP) and an analysis of radiosonde humidity profiles. They did not consider the cosmic factors.

Visa and Iivarinen (1997) presented NN based cloud classifier and showed its evolution capability. In their work, they used Advanced Very High Resolution Radiometer (AVHRR) images.

There are probabilistic approaches on cloud classification as well. Calbo et al. (2001) applied classical maximum likelihood method for clustering clouds. They used ground-based solar radiation measurements.

\section{Motivation}

Fuzzy inference is the process of formulating functions from a given input to an output using fuzzy logic. Fuzzy inference systems (FIS) are used to explain the specific methods of fuzzy inference (Matlab, 2005). A fuzzy inference system consists of inputs and their membership functions; output and its membership functions; and rules for the memberships.

A membership function (MF) is a curve that defines how each value in the input space is mapped to a membership value between 0 and 1 (Matlab, 2005). Thus, it specifies the degree to which a given input belongs to a set or is related to a concept (Matlab, 2005).

Depending on the methods for the fuzzy rules, FIS type is selected. The most common FIS types are Mamdanitype and Sugeno-type. Mamdani type is used widely by fuzzy system designers (Mamdani and Assilian, 1975; Matlab, 2005; Ozkok, 2005). In using FIS output as an input for NN, Mamdani-type inference is appropriate. In this work, METU-FIS uses Mamdani-type FIS. It is a type of fuzzy inference in which the fuzzy sets from the consequent of each rule are combined through the aggregation operator and the resulting fuzzy set is defuzzified to yield the output of the system (Matlab, 2005).

In this work, a data-driven model is developed, trained and applied for forecasting the \%CC and CTT, by considering the history of those meteorological variables; Cloud Optical Depth (COD); the Ionization (I) value that is formulized and computed by using CR data and CTT; SSN; temporal variables; and defuzified cloudiness. METU-FIS inputs are the temporal and spatial variables and the cut off rigidity. The defuzified cloudiness used in the input of the METU-FNN is the output of the METU-FIS.

The major advantage of the FIS is that it uses its inputs and the expert knowledge in order to obtain one of the inputs 
of the METU-FNN, i.e. defuzified cloudiness in our case. METU-NN is a purely data-driven Neural Network model. However, METU-FNN combines the advantages of the datadriven approach and the expert knowledge for higher performance. This is an important feature of the fuzzy systems, which increase the accuracy and sensitivity in the forecast values. To illustrate, the \% CC and CTT forecast performance of the METU-FNN could be compared with the performance of the METU Neural Network Model (METU-NN) without fuzziness. METU-NN has proven its success in forecasting nonlinear complex system parameters, i.e. ionospheric processes, agro-environmental processes and mechanical processes. Thus, METU-NN results can be used in order to evaluate METU-FNN results.

The forecast values at uniformly spaced grids over the region of interest are used for mapping. Bezier surfaces are applied for mapping purpose. They are not used in forecasting. The advantage of Bezier surfaces is the local control of the nonlinearity mapping with high accuracy when compared to Kriging method. The disadvantage of the Bezier surfaces is that they are not tolerant to missing data and non-uniform data. Since we used uniform and intense measurement data over the region of interest we found Bezier surfaces to be appropriate for mapping.

\section{Data}

The percent Cloud Coverage (\%CC) or the "Total Cloud Amount (TCA)" is the fraction of cloudiness. "Cloud Top Temperature (CTT)" is the temperature at the top of the highest clouds of interest. Cloud Optical Depth (COD) is the optical thickness of the clouds (http://isccp.giss.nasa. gov/products/browsed2.html). In this paper, the parameters to represent any probable climate change are chosen to be $\%$ CC, CTT, and COD. All the cloud data are smoothed by taking the 3-month running means.

"Cosmic Ray Flux (CRF)" and the monthly means of the "Sunspot Numbers (SSN)" data are considered as the Space Weather parameters, which influence the cloud formation. CRF and SSN data are obtained from the Climax CR database (ftp://ftp.ngdc.noaa.gov/STP/ SOLAR_DATA/COSMIC_RAYS/climax.tab) and from the SSN database (ftp://ftp.ngdc.noaa.gov/STP/SOLAR_DATA/ SUNSPOT_NUMBERS), respectively.

For the spatial coverage of the task, the cut off rigidity values are calculated via the cut off rigidity calculator java applet (ftp://nssdcftp.gsfc.nasa.gov/models/cosmic rays/cutoff_rigidity_sw/).

It is assumed that the variability of the CRF and CTT are related to the ionization produced by the CR's locally. Therefore, a statistical analysis is performed for the best-fit curve. Equation (1) is the analytical expression, which is used to obtain the amount of ionization in terms of 3-month running means of the CRF and the CTT for a specific month $k$.

$$
\begin{aligned}
I(k)= & 640\left(\frac{1}{2.2}(\ln (\operatorname{CRF}(k))-8.25)\right. \\
& \left.-0.003\left(\operatorname{CTT}(k)-\frac{\sum_{n=k-11}^{k} \operatorname{CTT}(n)}{12}\right)\right)+640
\end{aligned}
$$

The mean of the ionization values is found to be $640 \mathrm{~cm}^{-3}$, which checks with that of Usoskin et al. (2004).

The spatial and temporal data coverage include the region covering Europe, the Mediterranean Sea, and north Africa that is between $\left(22.5^{\circ} \mathrm{N} ; 57.5^{\circ} \mathrm{N}\right)$ and $\left(7.5^{\circ} \mathrm{W} ; 47.5^{\circ} \mathrm{E}\right)$ during the period between July 1983 and August 2000. The Earth's weather data are collected at every $5^{\circ}$ by $5^{\circ}$ latitude and longitude areas yielding 96 grid points.

The time period in between the years 1983 and 2000 cover two SSN peaks. The period can be investigated in two slots with similar solar activity, i.e. the first half in between 1983 and 1992 and the second half in between 1992 and 2000.

It is known that the CR shows an inverse relationship to the sunspot cycle because Sun's magnetic field is stronger during sunspot maximum and it shields the Earth from cosmic rays (http://www.ngdc.noaa.gov/stp/SOLAR/COSMIC RAYS/cosmic.html). We used the first slot data for training purpose and the second slot data for validation purpose.

\section{METU Fuzzy Neural Network Model (METU-FNN-M)}

One month in advance forecast values of \%CC and CTT are obtained by using the METU-FNN and the forecast maps are obtained by using Bezier surfaces (Senalp, 2007). To the best knowledge of the authors, it is the first time that a Fuzzy Neural Network with Bezier surface mapping technique is developed in order to forecast and map the parameters of such Near Earth space processes.

The forecast modeling process consists of (i) "training"; (ii) "validation within training; and (iii) "validation within operation" phases (Tulunay et al., 2004a). Unless otherwise stated the "validation within operation" will be referred as "operation".

Previously it has been demonstrated by the authors that data driven approaches such as the use of Neural Network (NN) based approaches are promising in modeling of ionospheric processes (Tulunay, 1991; Altinay et al., 1997; Y. Tulunay et al., 2000, 2001, 2004a, b; E. Tulunay et al., 2004; Radicella and Tulunay, 2004; Stamper et al., 2004; Tulunay et al., 2006).

In this work, the \%CC and CTT forecast model consists of one Mamdani-type FIS block (METU-FIS) and one FNN block (METU-FNN). Figure 1 shows the connections of the 


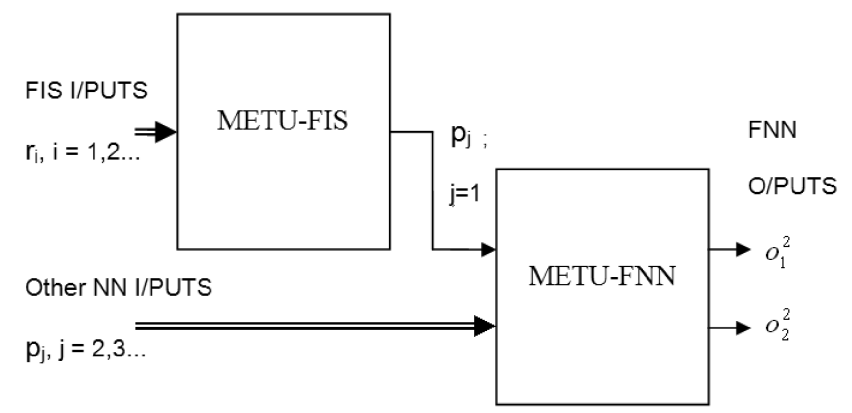

Fig. 1. METU Fuzzy Neural Network Model (METU-FNN-M) block diagram having the METU Fuzzy Inference System (METUFIS) and the METU-FNN blocks.

METU-FIS and METU-FNN blocks. The model has 96 submodules corresponding to 96 grid locations. The METUFNN-M has been developed on Matlab platform.

The architectures of the METU-FIS and METU-FNN blocks are given in Fig. 2 and Fig. 3, respectively. METUFIS is the block that converts its inputs to fuzzy variables and then provides a defuzzified cloudiness variable, which is one of the inputs in the METU-FNN. Thus, METU-FIS uses the expert information in the model and it is connected to the METU-FNN. METU-FNN is the Artificial Intelligent (AI) block having data-driven learning capability. It gives the $\% \mathrm{CC}$ and CTT forecast values at its output layer.

The detailed inner structure of the METU-FIS is illustrated schematically in Fig. 2. In this figure, the input parameters to the METU-FIS are the time (year number and season information); geographic coordinates (latitude and longitude); and cut off rigidity. Membership functions or logical rules to employ in the Mamdani type METU-FIS are established for these input parameters by studying the seasonal, geographical and cut off rigidity dependences of $p_{1}$ (cloudiness). Lower \%CC values are observed during summer in Northern Hemisphere, and \%CC increases for the northern geographical latitudes. These are the two major factors affecting $\% \mathrm{CC}$. There are also other factors. For the region of interest, in general, as geographical longitude increases \%CC values slightly decrease. Higher the cut-off rigidity factor, lower the $\mathrm{CRF}$ penetrate, thus lower the \%CC values are. One minor effect for the \%CC values is the year of interest. In this era, in long term, \%CC values are reduced. With those properties, fuzzy rules and membership functions for the METU-FIS inputs and output are assigned. The output of the METU-FIS or the first input of the METU-FNN block is the defuzzified form of $p_{1}$ (cloudiness).

Figure 3 illustrates the detailed inner structure of the METU-FNN schematically. Among the various NN structures the best configuration is found to be the one with one hidden layer. In this work, 20 neurons were used in the hidden layer. There are 16 inputs, 20 hidden neurons and 2 outputs in the feed-forward structure.

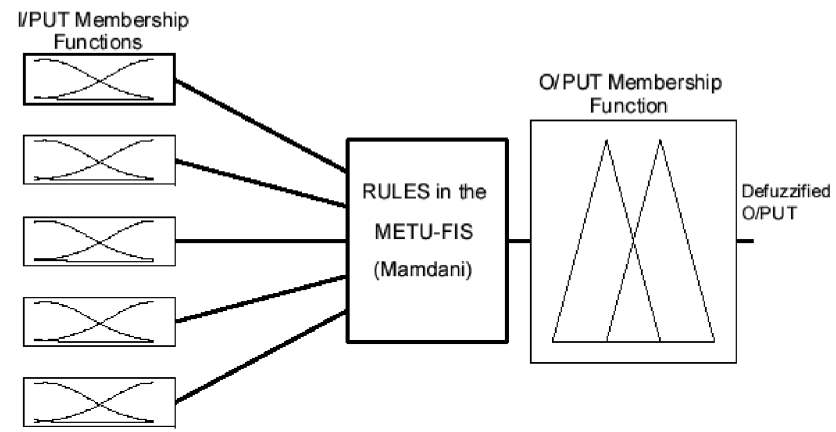

Fig. 2. Structure of the METU Fuzzy Inference System.

Table 1 shows all the input parameters to METU-FNN and the outputs of METU-FNN. The inputs of the METUFNN are the defuzified cloudiness, which is the METU-FIS output; trigonometric components of the temporal variables; the meteorological variables: \%CC, CTT and Cloud Optical Depth (COD); the Ionization (I) value that is computed by using CR data and CTT; SSN; and the history of the meteorological variables. First, second and relative difference values of the system parameters are three major history inputs in the METU-FNN. First and second difference values in discrete time domain correspond to the first and second time derivatives in continuous time domain. They provide dynamic information for the processes. The physical impact of them can be illustrated in any dynamic problem of interest, (e.g. velocity and acceleration in mechanical systems). Relative difference provides extra information by normalizing the first difference values with respect to the initial value in order to avoid scaling problems in modeling. To justify, if the initial parameter value is small, then a considerable amount of change of a parameter is to be treated accordingly. However, if the initial value is large, the same amount of change of the parameter may be of less importance. Relative difference is appropriate for such an evaluation.

The periods of data coverage in "training" and "operation" phases are denoted in Table 2.

The METU-FNN module needs a large amount of data and the nonlinear inherent process is to be learned as fast as possible during the "training" phase. Therefore, the LevenbergMarquardt Backpropagation algorithm is chosen to be the most convenient one (Hagan and Menhaj, 1994; Haykin, 1999).

As the training process advances, the training error starts to decrease, and it eventually goes to zero. Zero error corresponds to memorization state. Memorization means the loss of the generalization capability of the Neural Network. To prevent memorization, when the gradient of the error in the "validation within training" phase becomes near zero training is terminated. The model is then ready for its actual use in the forecasting operation. In the operation mode the validation data are used for calculating the errors, point by point, to measure the performance of the model. 


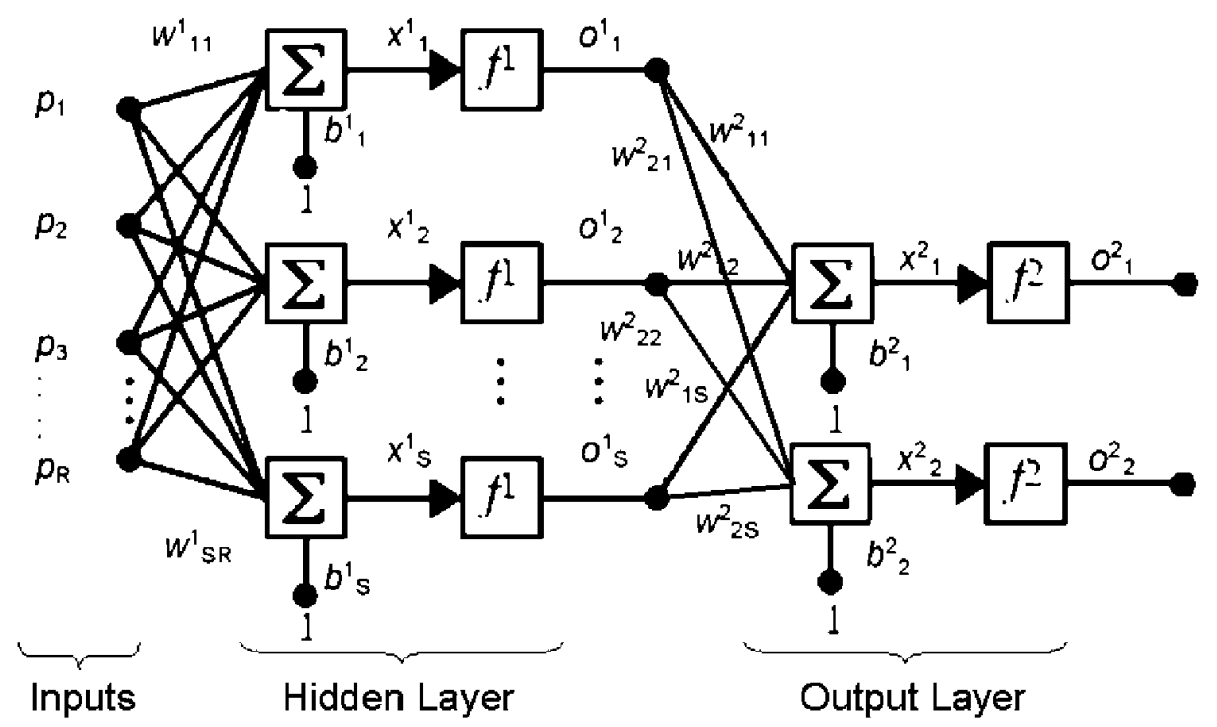

Fig. 3. Architecture of the METU Fuzzy Neural Network (METU-FNN) module where $p_{1}$ is defuzified input.

Table 1. Inputs and outputs of the METU-FNN.

\begin{tabular}{lll}
\hline I/PUT number & I/PUT & O/PUT \\
\hline$p_{1}$ & defuzzified cloudiness & \\
$p_{2} ; p_{3}$ & $\sin (2 . \pi \cdot \mathrm{k} / 12) ;-\cos (2 . \pi \cdot \mathrm{k} / 12)$ & \\
$p_{4}$ & $f_{1} \equiv \% \mathrm{CC}(\mathrm{k})$ & $\% \mathrm{CC}(\mathrm{k}+1)$ \\
$p_{5}$ & $f_{2} \equiv \mathrm{CTT}(\mathrm{k})$ & and \\
$p_{6}$ & $f_{3} \equiv \mathrm{COD}(\mathrm{k})$ & $\mathrm{CTT}(\mathrm{k}+1)$ \\
$p_{7}$ & $I(k)$ & \\
$p_{8}$ & $\mathrm{SSN}(\mathrm{k})$ & \\
$p_{9} ; p_{10} ; p_{11}$ & $\Delta_{1}\left(f_{i=1,2,3}(k)\right)=f_{i=1,2,3}(k)-f_{i=1,2,3}(k-1)$ & \\
$p_{12} ; p_{13} ; p_{14}$ & $\Delta_{2}\left(\mathrm{f}_{i=1,2,3}(k)\right)=\Delta_{1}\left(f_{i=1,2,3}(k)\right)-\Delta_{1}\left(f_{i=1,2,3}(k-1)\right)$ & \\
$p_{15} ; p_{16}$ & $R \Delta\left(f_{i=1,2}(k)\right)=\Delta_{1}\left(f_{i=1,2}(k)\right) / f_{i=1,2}(k)$ & \\
\hline
\end{tabular}

\section{Mapping by using Bezier surfaces}

As explained in the previous sections, for each grid, the input parameters to the METU-FIS are the time (year number and season information); geographic coordinates (latitude and longitude); and cut off rigidity. The spatial information has been embedded in the METU-FIS. Thus, the METU-FNN \% CC and CTT forecast outputs for each grid have spatial information even before the mapping process.

In addition, local spatial correlations are considered by applying Bezier surfaces in the mapping process. Various computer graphics applications use Bezier surfaces in surface mapping since the approach is very sensitive in local control (Rogers and Adams, 1990).

The Bezier surfaces have been used for mapping of some Near Earth Space parameters by the authors previously (Tulunay et al., 2006).
Table 2. Organization of data.

\begin{tabular}{cl}
\hline Phase & Period \\
\hline Training & August 1984 to August 1992 \\
Operation & September 1992 to August 2000 \\
\hline
\end{tabular}

Equation (2) is employed in computing the \%CC or CTT forecast values at any location on the map by using Bezier surfaces.

$$
f(u, w)=\sum_{i=0}^{n} \sum_{j=0}^{m} B_{i+1, j+1} . J_{n, i}(u) . K_{m, j}(w)
$$



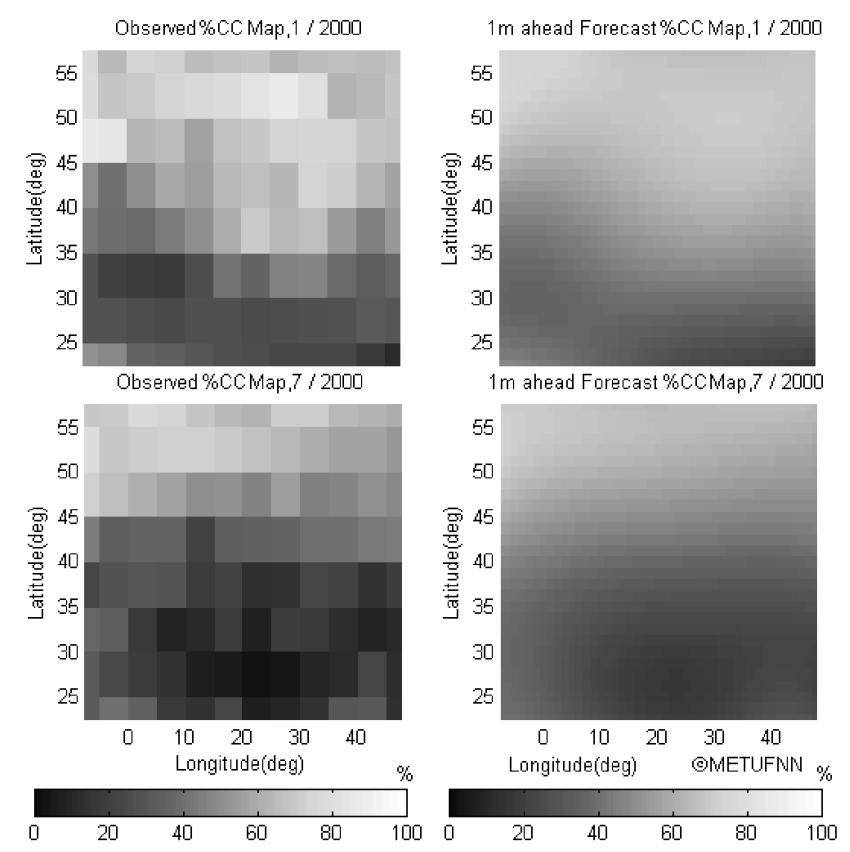

Fig. 4. Observed and 1 month ahead Forecast \%CC maps of January and July 2000.

where $J$ and $K$ are the Bernstein basis functions as denoted in Eq. (3).

$$
\begin{aligned}
J_{n, i}(u) & =\left(\begin{array}{c}
n \\
i
\end{array}\right) u^{i}(1-u)^{n-i} \\
K_{m, j}(w) & =\left(\begin{array}{c}
m \\
j
\end{array}\right) w^{j}(1-w)^{m-j}
\end{aligned}
$$

The elements of the matrix B are the METU-FNN \%CC and CTT outputs for the grids. The 96 grids are formed as $(n+1=12$ longitude) by $(m+1=8$ latitude $)$ segments. In Eqs. (2) and (3), $u$ and $w$ denote the normalized values of longitude and latitude, respectively.

\section{Results}

In the "operation" mode, the 96 modules of the METU-FNN yield the 96 one month in advance values of the Percent Cloud Coverage (\%CC) and Cloud Top Temperature (CTT). These forecast values are then used in the Bezier surface equations to produce maps.

The maps of the \%CC and CTT are constructed throughout the spatial and temporal "operation" coverage of interest. A video presentation is available at the www.ae.metu.edu.tr/ $\sim$ cost. Some typical examples of such maps are presented in Figs. 4 and 5. They are the maps to demonstrate the forecast and mapping performance of the METU-FNN-M for forecasting \%CC and CTT.

Figures 6 and 7 exhibit how the forecast values of \%CC and CTT follow the inherent nonlinearities of the observed
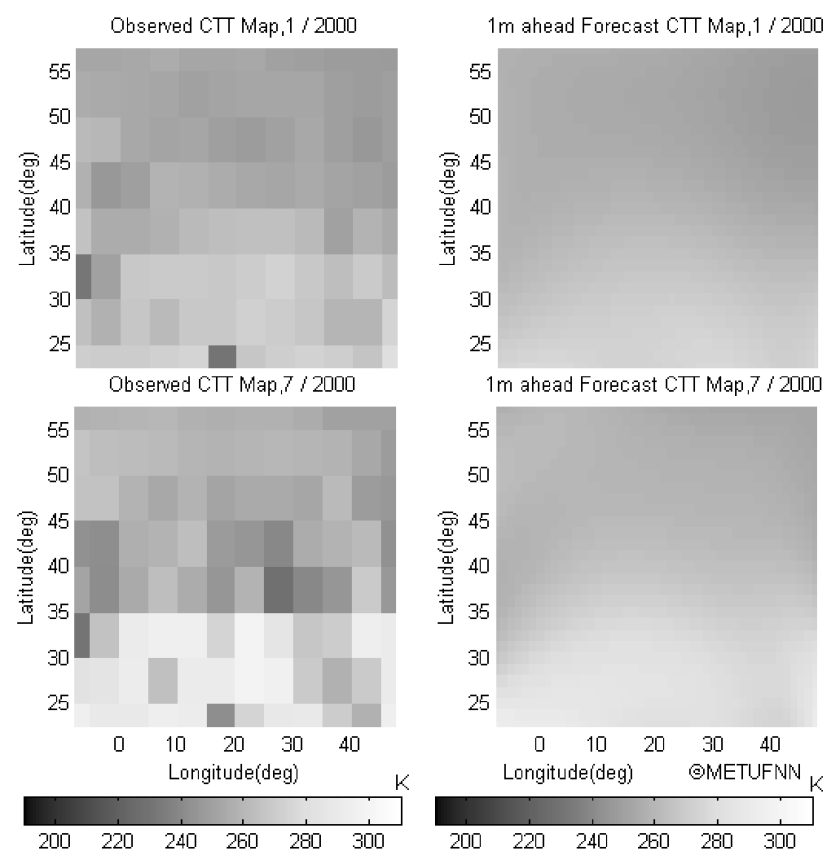

Fig. 5. Observed and 1 month ahead Forecast CTT maps of January and July 2000.

\%CC and CTT, respectively, on the average. Superimposed on the top of the figures are their absolute error variations. In these figures, the observed data are superimposed by the corresponding forecast values at a randomly chosen grid location of $\left(37.5^{\circ} \mathrm{N} ; 27.5^{\circ} \mathrm{E}\right)$ during the operation period of September 1992 to August 2000.

The performance measure of the forecast operation is expressed in terms of absolute error values computed at each one of the 96 grid locations. Figures 8 and 9 are illustrating the error maps.

Figures 10 and 11 are the scatter diagrams of the observed and forecast \%CC; and the observed and forecast CTT at the sample grid location of $\left(37.5^{\circ} \mathrm{N} ; 27.5^{\circ} \mathrm{E}\right)$ during September 1992 to August 2000, respectively.

As it is shown, observed and forecast \%CC and CTT variations are coherent. The forecasting errors between observed and forecast \%CC and CTT are small and the best-fit lines in the scatter diagrams have slopes close to one. Thus, the model reached correct operating point. The correlation coefficients between observed and forecast \%CC and CTT maps are very close to one and the deviations from straight lines in the scatter diagrams are small. Thus, the model learned the inherent nonlinearities of the process of interest.

METU-NN results can be used in order to evaluate METU-FNN results further. The average absolute error values in forecasting \%CC by METU-FNN and by METU$\mathrm{NN}$ are $4.26 \%$ and $4.31 \%$ respectively. The average absolute error values in forecasting CTT by METU-FNN and by METU-NN are $3.38 \mathrm{~K}$ and $3.40 \mathrm{~K}$, respectively. The average 


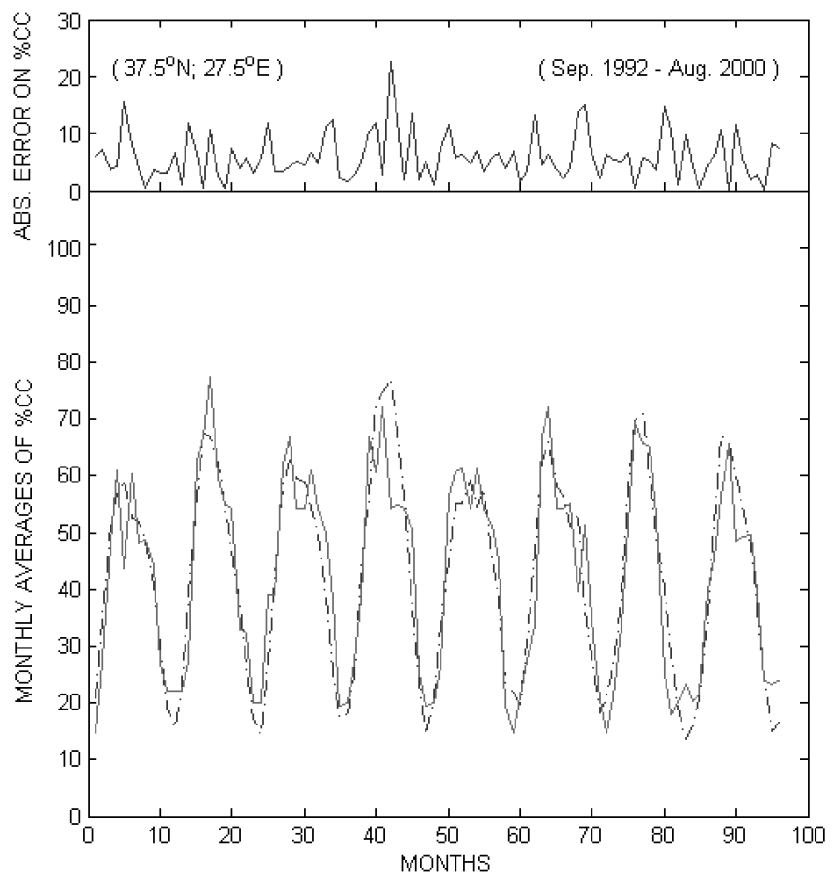

Fig. 6. Observed monthly averages of the \%CC values (dashed) and superimposed are the one-month in advance forecast \%CC values (solid) versus time. The absolute error values on the computed parameters versus time are shown in the top panel.

cross correlation value for the forecast results by METUFNN and for the observed values is $1 \%$ higher than the ones by METU-NN.

\section{Conclusions}

In this paper, the Percent Cloud Coverage (\%CC) and Cloud Top Temperatures (CTT) are forecast one month ahead of time at 96 grid locations. The probable influence of Cosmic Rays (CR) and Sunspot Numbers (SSN) on cloudiness is considered.

Fuzzy Neural Network Model, METU-FNN-M is constructed for the task. Bezier surfaces are used in obtaining the forecast \%CC and CTT maps over a region including Europe, the Mediterranean Sea and North Africa. The results are exhibited as maps and individual curves.

Forecast \%CC and CTT maps are obtained by using METU-FNN-M in the operation time of interest, September 1992-August 2000. Forecasting errors are small. This fact is the indication of the system reaching the correct operating point within training. In other words, the system is prevented to reach local minima of the error cost function and it is succeeded to reach the global minimum. The correlation coefficients between the observed and forecast values are very close to one, which means that the METU-FNN-M learned the shape of the inherent nonlinearities as noted by the best-

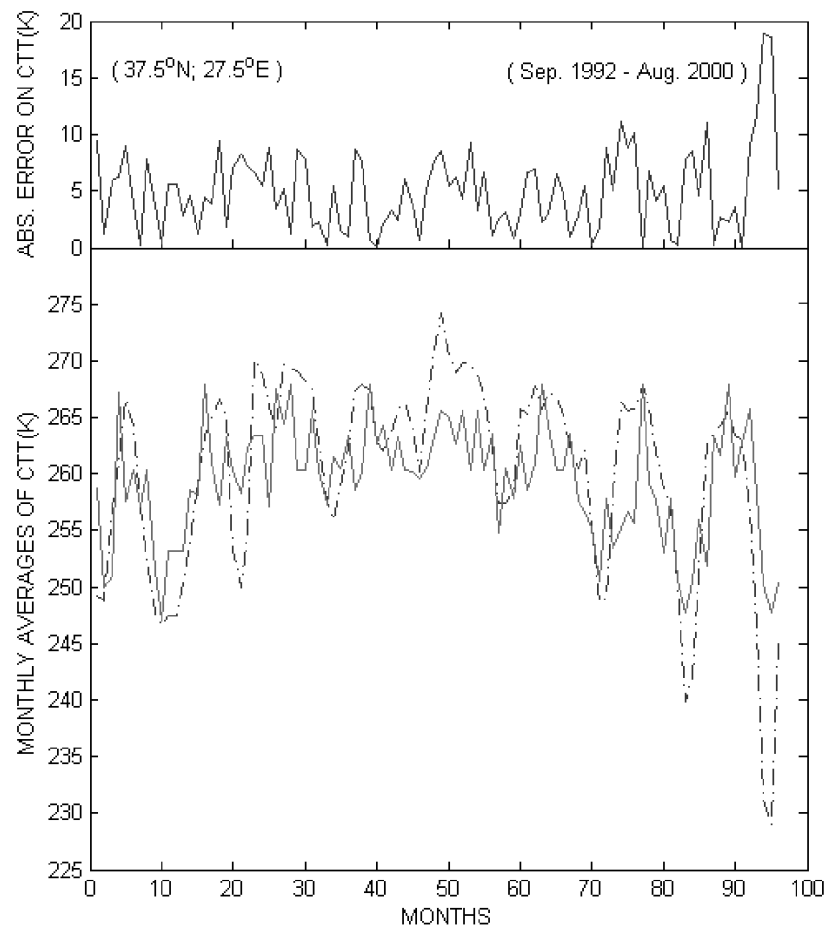

Fig. 7. Observed monthly averages of the CTT values (dashed) and superimposed are the one month in advance forecast CTT values (solid) versus time. The absolute error values on the computed parameters versus time are shown in the top panel.

fit line in the scatter diagrams. As a conclusion, it is shown that properly constructed Fuzzy Neural Network based systems, trained and tested with properly organized data including the ones organized by constructing intelligent fuzzy inference systems are promising in modeling the complex nonlinear processes, such as the variability of the \%CC and CTT values. The forecast mapping performance is within operational tolerance (private communication: M. Yayvan, Turkish State Meteorological Service, Ankara, Turkey, 2007).

The heat transports are caused by dynamic complex systems of different sources. Thus, the performance of forecasting CTT is lower than the performance of forecasting \%CC. Within the dynamics, solar radiation is converted into heat and "heat" radiation, water vapor and the circulations of the atmosphere and ocean by the climate system (Zhang et al., 2004). The optical properties of the cloud particles, optical depth, cloud formation level and dynamical processes affect the CTT variation.

Clouds play important role on the Earth's atmosphere in terms of the radiation balance. It has cooling and warming effects. The cooling effect takes place as a result of the reflection of the short wave radiation coming to atmosphere. The warming effect is due to the absorption of the long wave radiation emitted from the Earth's surface. 


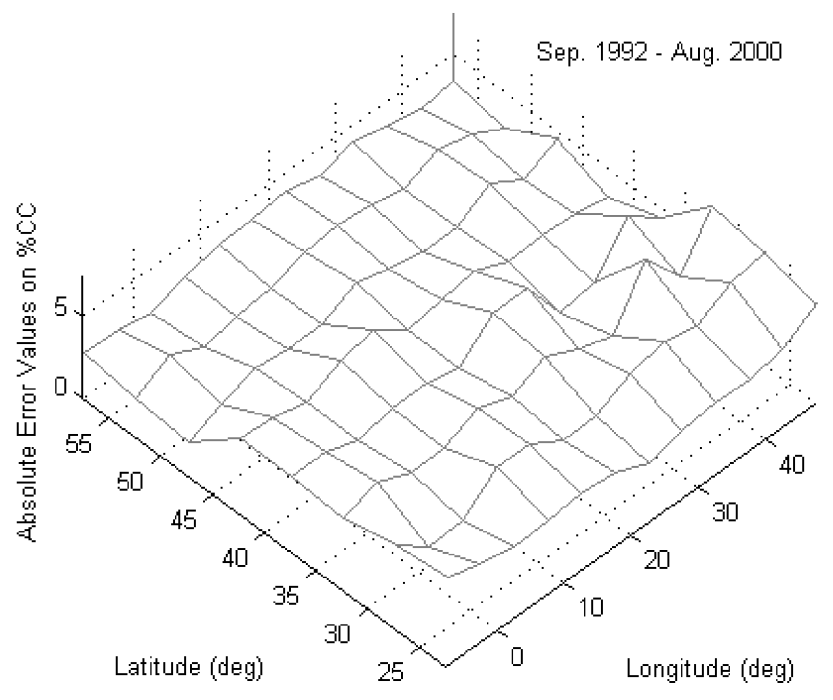

Fig. 8. The map of the absolute error values on the \%CC versus latitude and longitude grids.

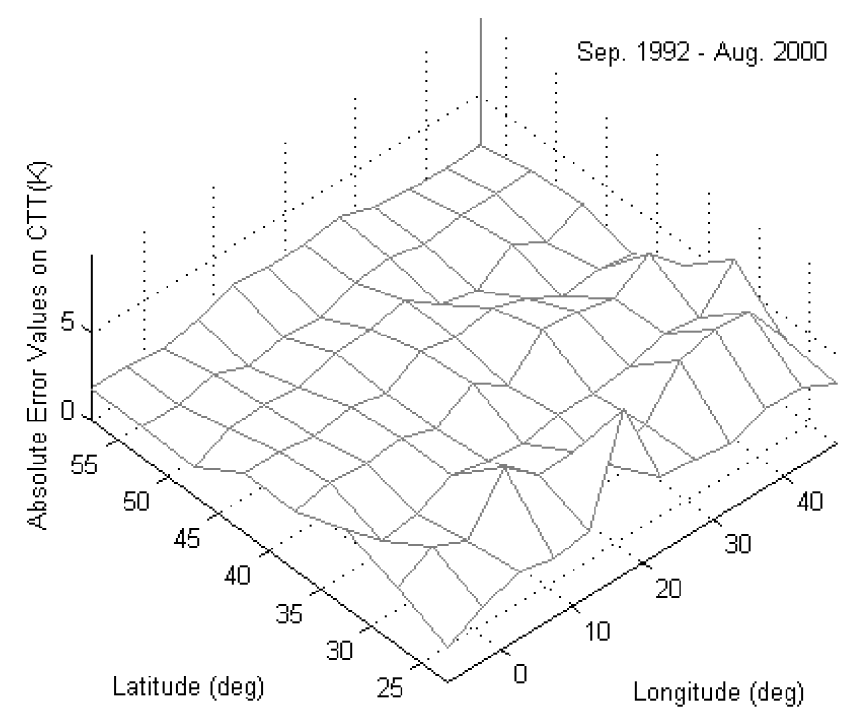

Fig. 9. The map of the absolute error values on the CTT versus latitude and longitude grids.

These effects vary according to the height and type of the cloud, amount of radiation reflected from the cloud, and temporal and microphysical characteristics of the cloud.

The formation of the clouds can be estimated by several meteorological parameters such as moisture, temperature, and other parameters related with the atmospheric dynamics. The required physical and dynamical factors are the amount of water vapor and the cooling process, respectively. These factors allow the condensation, large scale systematic motions, rising motions over the depression regions, uplift along weather fronts and low-level convergence areas, medium and small-scale convection, and orographic rising.

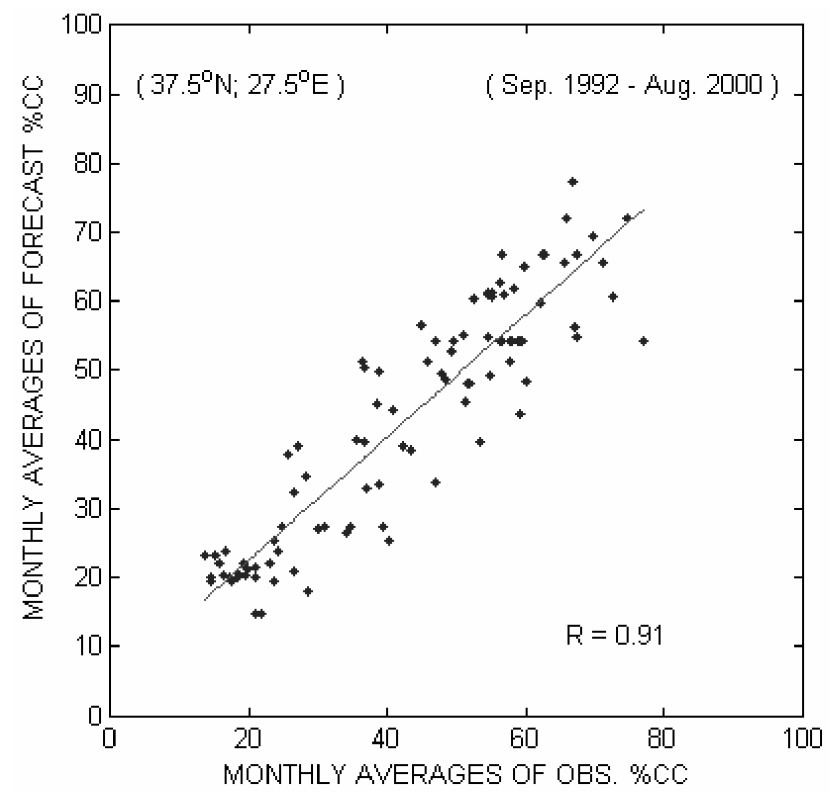

Fig. 10. The scatter diagram of the one-month in advance forecast $\%$ CC values versus the observed \%CC values and the best-fit line. $R$ is the cross correlation coefficient between the forecast and the observed values.

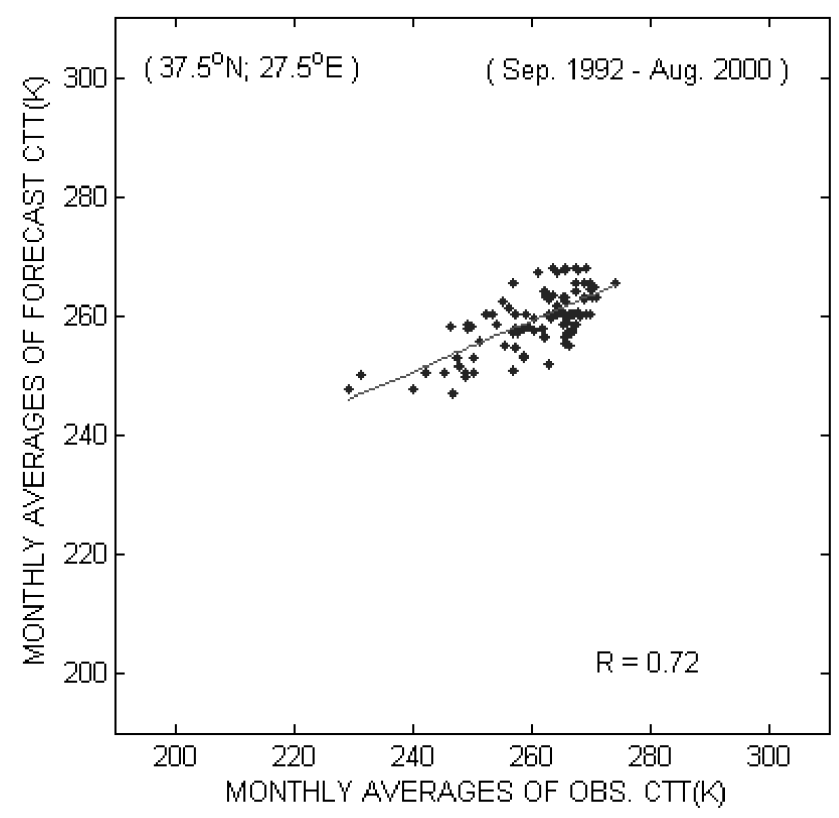

Fig. 11. The scatter diagram of the one-month in advance forecast CTT values versus the observed CTT values and the best-fit line. $R$ is the cross correlation coefficient between the forecast and the observed values.

As a result of these physical and dynamical processes, clouds have different types of microphysical properties at different levels. For example, the low level clouds are the clouds, which have high temperature at the cloud top and high optical thickness. 
The optical thickness of the clouds depends on the dimension of the cloud, factors affecting the distribution, and the vertical temperature profile (Akcan, 2004).

Aerosols affect the cloud formation process. The relation between the cosmic rays and the clouds comes from the effect of the ions on the cloud microphysics. This mechanism can be described as the growing of the condensation nuclei as a result of the combination of the ions with the atmospheric aerosols. The higher the number of ions, the higher the environmental water vapor concentration is. Change in the amount of cosmic ray flux affects the effectiveness of the charged aerosols around the clouds. As a result of this, microphysical processes, which include the aerosols and cloud droplets, can be affected (Swensmark, 2000).

There are uncertainties in climate forcing research, because of the lack of detailed knowledge of the optical properties of aerosols. Works on characterization of aerosol will help refine aerosol optical models and reduce uncertainties in satellite observations of the global aerosol and in modelling aerosol impacts on climate (Dubovik et al., 2002).

In this work, in order to show the generalization capability, long-term cloud analysis has been performed on a region showing differences in terms of atmospheric activity. To illustrate, one of the grids on the region is at $\left(37.5^{\circ} \mathrm{N}, 27.5^{\circ} \mathrm{E}\right)$. In general, this sample grid location is under the effect of the Mediterranean frontal zone and temporary cyclones are active and effective in winters. These conditions are the basis for the rising motions along the frontal zone and cyclone for the formation of the clouds. On the other hand, the vertical convective motions depending on the land warming over this sample grid location dominate the summer season.

Consequently, vertical motions depending on the different mechanisms, cloud amount, cloud type and level and the cloud top temperature show differences temporally and spatially. However, the effects of the CR and SSN are general.

In the METU-FNN-M, both CR Flux and SSN reflect the influence of Space Weather on general planetary situation; but other parameters in the inputs of the model reflect local situation. Thus global and local parameters of the process are considered. This is the main cause why the correlations between the forecast and observed parameters are very promising.

Quantification of physical mechanisms, which causally link Space Weather to the Earth's Weather and climate, in order to reduce the uncertainties in the level of scientific understanding of aerosols and solar forcing on the global climate system, has been a challenging task. This has been specified in the Intergovernmental Panel on Climate Change (IPCC) 2001 (private communication: M. Fullekrug, Coordinator of the ESF Network: Space Weather and the Earth's Weather and Climate (SPECIAL), Frankfurt, Germany, 2002). In this connection, to study the probable effect of the CR and SSN on the \%CC/CTT, one needs to include, at least few solar cycle long data. For such an investigation, the model developed here would be useful since the CR Flux as given one of the inputs to the model improved the performance of the model in terms of smaller errors in the forecast of \%CC and CTT values. It is hoped that this model would be used in parallel with mathematical models based on the first physical principles. The model contributes to the cloud formation process, in particular, its dependence on CR Fluxes.

The system developers may use the one-month in advance forecast values of the model as inputs to other models, which forecast some other local or global parameters in order to test the hypothesis on possible link(s) between Space Weather and the Earth's Weather and climate.

It is believed that this is the first forecast mapping application of a Fuzzy Neural Network and Bezier surface fitting on the \% CC and CTT values over Europe.

Acknowledgements. EU COST 296: Mitigation of Ionospheric Effects on Radio Systems; (TÜBITAK/105Y003) and the EU COST 724: Developing the Scientific Basis for Monitoring, Modeling and Predicting Space Weather Actions; (TÜBITTAK/103Y189) are acknowledged for the partial support.

Topical Editor F. D'Andrea thanks two anonymous referees for their help in evaluating this paper.

\section{References}

Akcan, E.: Güneş Lekeleri Çevrim Süreci İçerisinde Kozmik Işınların Yeryüzü İklim-Bulut Örtüsü ile olan İlişkisinin İncelenmesi, MSc. Thesis, supervisor: Menteş, Ş. S., cosupervisor: Tulunay, Y., Inst. of Science and Technology, İÜ̈, 2004.

Altinay, O., Tulunay, E., and Tulunay, Y.: Forecasting of ionospheric critical frequency using neural networks, Geophys. Res. Lett., 24(12), 1467-1470, 1997.

Calbo, J., Gonzales, J-A., and Pages, D.: A Method for SkyCondition Classification from Ground-Based Solar Radiation Measurements, J. Appl. Meteorol., 40, 2193-2199, 2001.

Carslaw, K. S., Harrizon, R. G., and Kirkby, J.: Cosmic rays, clouds, and climate, Science, 298(5599), 1732-1737, 2002.

Dorman, L. I.: Cosmic Rays in the Earth's Atmosphere and Underground, Springer, ISBN: 978-1-4020-2071-1, 2004.

Dubovik, O., Holben, B., Eck, T. F., Smirnov, A., Kaufman, Y. J., King, M. D., Tanre, D., and Slutsker, I.: Variability of Absorption and Optical Properties of Key Aerosol Types Observed in Worldwide Locations, J. Atmos. Sci., 59, 590-608, 2002.

Hagan, M. T. and Menhaj, M. B.: Training Feedforward Networks with the Marquard Algorithm, IEEE T. Neural Networ., 5(6), 989-993, 1994.

Haykin, S.: Neural Networks: A Comprehensive Foundation, 2nd ed., 2, 10, 21-22, 83-84, 169, 215, Prentice-Hall, Inc., New Jersey, USA, 1999.

Mamdani, E. H. and Assilian, S.: An experiment in linguistic synthesis with a fuzzy logic controller, Int. J. Man Mach. Stud., 7(1), $1-13,1975$.

Matlab: v7.0 R14 Documentation, The MathWorks, Inc., 2002.

Ozkok, Y.: Web Based Ionospheric Forecasting Using Neural Network and Neurofuzzy Models, MS Thesis, supervisor: Tulunay, E., co-supervisor: Tulunay, Y., Dept. of Electrical and Electron- 
ics Eng., Middle East Technical University, Ankara, Turkey, July 2005.

Palle, E., Butler, C. J., and O'Brien, K.: The possible connection between ionization in the atmosphere by cosmic rays and low level clouds, J. Atmos. Sol.-Terr. Phys., 66, 1779-1790, 2004.

Radicella, S. M. and Tulunay, E.: Space plasma effects on Earthspace and satellite-to-satellite communications: Working Group 4 overview, Ann. Geophys.-Italy, 47(2/3), 1279-1283, 2004.

Rogers, D. F. and Adams, J. A.: Mathematical Elements for Computer Graphics, 2nd ed., 379-477, McGraw-Hill, Inc., New York, USA, 1990.

Rossow, W. B., Zhang, Y., and Wang, J.: A Statistical Model of Cloud Vertical Structure Based on Reconciling Cloud Layer Amounts Inferred from Satellites and Radiosonde Humidity Profiles, J. Climate, 18, 3587-3605, 2005.

Senalp, E. T.: Cascade Modeling of Nonlinear Systems, PhD Thesis, supervisor: Tulunay, E., Dept. of Electrical and Electronics Eng., Middle East Technical University, Ankara, Turkey, August 2007.

Stamper, R., Belehaki, A., Buresova, D., Cander, Lj. R., Kutiev, I., Pietrella, M., Stanislawska, I., Stankov, S., Tsagouri, I., Tulunay, Y. K., and Zolesi, B.: Nowcasting, forecasting and warning for ionospheric propagation: tools and methods, Ann. Geophys.Italy, 47(2/3), 957-983, 2004.

Svensmark, H.: Cosmic Ray and Earth's Climate, Space Sci. Rev., 93, 155-166, 2000.

Todd, M. C. and Kniveton, D. R.: Changes in cloud cover associated with Forbush decreases of galactic cosmic rays, J. Geophys. Res.-Atmos., 106(D23), 32 031-32 041, 2001.

Tulunay, E.: Introduction to Neural Networks and their Application to Process Control, in: Neural Networks Advances and Applications, edited by: Gelenbe, E., 241-273, Elsevier Science Publishers B.V., North-Holland, 1991.

Tulunay, E., Senalp, E. T., Cander, Lj. R., Tulunay, Y. K., Bilge, A. H., Mizrahi, E., Kouris, S. S., and Jakowski, N.: Development of algorithms and software for forecasting, nowcasting and variability of TEC, Ann. Geophys.-Italy, 47(2/3), 1201-1214, 2004.
Tulunay, E., Senalp, E. T., Radicella, S. M., and Tulunay, Y.: Forecasting Total Electron Content Maps by Neural Network Technique, Radio Sci., 41(4), RS4016, American Geophysical Union, Washington, USA, 2006.

Tulunay, Y., Tulunay, E., Senalp, E. T., and Ozkaptan, C.: Neural Network Modeling of the Effect of the IMF Turning on the Variability of HF Propagation Medium, AP 2000, Millennium Conference on Antennas \& Propagation, ICAP\&JINA, 132, 914 April 2000, Davos, Switzerland, 2000.

Tulunay, Y., Tulunay, E., and Senalp, E. T.: An Attempt to Model the Influence of the Trough on HF Communication by Using Neural Network, Radio Sci., 36(5), 1027-1041, 2001.

Tulunay, Y., Tulunay, E., and Senalp, E. T.: The Neural Network Technique-1: A General Exposition, Adv. Space Res., 33(6), 983-987, 2004a.

Tulunay, Y., Tulunay, E., and Senalp, E. T.: The Neural Network Technique-2: An Ionospheric Example Illustrating its Application, Adv. Space Res., 33(6), 988-992, 2004b.

Tulunay, Y., Senalp, E. T., Oz, S., Dorman, L. I., Tulunay, E., Mentes, S. S., and Akcan, M. E.: Forecasting Total Cloud Amount Maps and Cloud Top Temperature Maps by using Fuzzy Neural Networks and Bezier Surfaces, The 9th WMO Scientific Conference on Weather Modification, 22-24 October 2007, Antalya, Turkey, 2007.

Usoskin, I. G., Gladysheva, O. G., and Kovaltsov, G. A.: Cosmic ray induced ionization in the atmosphere: spatial and temporal changes, J. Atmos. Sol.-Terr. Phys., 66(18), 1791-1796, 2004.

Visa, A. and Iivarinen, J.: Evolution and Evaluation of a Trainable Cloud Classifier, IEEE T. Geosci. Remote, 35(5), 1307-1315, 1997.

Zhang, Y. Rossow, W. B., Lacis, A. A., Oinas, V., and Mishcenko, M. I.: Calculation of radiative fluxes from the surface to top of atmosphere based on ISCCP and other global data sets: Refinements of the radiative transfer model and the input data, J. Geophys. Res., 109, D19105, doi:10.1029/2003JD004457, 2004. 